el diseño de zapatillas, instalaciones de arte o campañas publicitarias para MTV.

Tanto Love como Tronic Studio encaran -tras el discurso de la versatilidad de servicios y la combinación de experiencias- casi literalmente, la pregunta por la promiscuidad: existe una clara postura que integra un posicionamiento ante el mercado, ante la tecnología y lo mediático. Una definición de la arquitectura como una disciplina que, desde otro enfoque, puede dar cabida a proyectos diversificados.

La máquina de los deseos... hacia... dónde?4 / "Se vende la marca y la marca es arquitectura"... "(los arquitectos) por eso están dando el último aliento, incorporándose a cualquier instancia (sólo falta imaginar a Boza en 'Cocinando con Mónica')"... "Toda una crisis existencial. Pero Arquitectura Deseable ${ }^{\circledR}$ es una manera de precipitar la acción, el consumo de arquitectura es parte del mercado"... "No sólo la arquitectura comercial es un producto de consumo: se consumen formas de vida, se consumen 'centros para la difusión de la cultura aymara', se consume 'la inmortalidad del cangrejo, versión Eisenman'... en las facultades, se consumen ediciones piratas del Mutaciones"... "La extensión de la arquitectura no sólo debe ser a partir de bacer más tipos de cosas (o tipos de casas), sino que su llegada debe poseer esta cualidad. Las tácticas mediáticas ya participan en la arquitectura, en la gestión de los proyectos, en los discursos de los arquitectos, en la carrera de los arquitectos, pero como un pegoteo ajeno y medio bastardo"... "La publicidad como substancia, como medio de difusión, de comunicación".

Ceder desde la arquitectura para dar paso a la arquitectura. Para este contexto, dogmatizar restringe. $\mathrm{Y}$ ése es el horizonte que no quiere perderse. (Des)variar, y seguir siendo. ARQ

Bibliografía: AA.VV.; Verb processing, architecture boogazine. ACTAR Ediciones, Barcelona, 2001. / Baudrillard, Jean; La transparencia del mal. Editorial Anagrama, Barcelona, 1991. / Costa, Joan y Moles, Abraham; Publicidad y diseño. Ediciones Infinito, Buenos Aires, 1999. / García Adánez, Isabel y Bravo De La Varga, Roberto (traductores); Nueva arquitectura alemana. Una modernidad reflexiva. Ministerio de Fomento, Madrid, 2002. / Leach, Neil; La an-estética de la arquitectura. Editorial Gustavo Gili, Barcelona, 2001. / Ockman, Joan; "El hombre del $¥ € \$$, de Harvard a Prada, Koolhass en clave de consumo". En Arquitectura Viva $\mathrm{N}^{\circ}$ 83, Arquitectura Viva S.L., Madrid, 2002. / Pérez Arnal, Ignasi; "Se vende el arquitecto: el arte de la ventaja". En revista Quaderns d' arquitectura i urbanisme $\mathrm{N}^{\circ} 221$, C.O.A.C., Barcelona, 1997. / Venturi, Robert; Izenour, Steven y Scott Brown, Denise; Aprendiendo de Las Vegas, el simbolismo olvidado de forma arquitectónica. Original de 1972. Editorial Gustavo Gili, Barcelona, 1998.
Cuatro escuelas de arquitectura

\author{
Sobre la formación de los arquitectos \\ Fuan Ignacio Baixas. Director de la Escuela de \\ Arquitectura, Universidad Católica de Chile
}

Al reflexionar sobre la formación de los arquitectos surgen dos preguntas genéricas: La primera pregunta es: ¿Qué del oficio de la arquitectura deben aprender los estudiantes durante sus estudios universitarios? Se debe considerar por una parte que la complejidad del oficio exige un aprendizaje que dura toda la vida y por otra que en Chile la formación universitaria no sólo entrega un grado académico sino también un título profesional.

La segunda pregunta es: ¿Cómo aprenderlo, teniendo en cuenta que es un oficio que complementa la utilidad de los oficios técnicocientíficos con la gratuidad de los oficios artísticos?

En respuesta a la primera pregunta, creo que lo fundamental de la formación universitaria de los arquitectos consiste en la adquisición de una ética que tiene que ver con la creatividad y una voluntad de actuar sobre la realidad.

La ética de la creatividad y la voluntad de lo real son dos temas que en forma tácita se han ido conformando en nuestra escuela a lo largo de años. Temas que intentamos preservar $y$ consolidar en un espíritu de escuela.

El primero de ellos tiene que ver con el hecho de que una obra de arquitectura debe responder cada vez a un encargo u origen circunstancial, con su propio tiempo, lugar y usuario y por lo tanto es una obra original. Tal originalidad tiene por objeto construir un futuro (cultura) y pertenece por lo tanto, necesariamente, al ámbito de la creatividad.

Tal originalidad sin embargo, debe redundar en una obra habitable y por lo tanto está obligada a considerar los múltiples aspectos que tal habitabilidad exige, sin dejar ninguno de lado. Esto es difícil en un mundo en que las acciones tienden a especializarse y a descartar asuntos cruciales.

Un mundo que, al decir de Heidegger, está amenazado por el peligro metafísico de la esencia de la técnica moderna en que la producción ya no se centra en el sentido originario de poiesis que le dieron los griegos sino más bien en la explotación de recursos planetarios con todo lo que ello conlleva.

Un mundo en que nuestra universidad, con una 
clara vocación ética cristiana, se autoimpone -por sobre la ética básica de la justicia-, una ética de la caridad a lo cual nuestra escuela agrega como tema ético un desarrollo del mayor talento que el Creador nos entrega, el de la creatividad destinada a la producción de belleza, de utilidad, de temperie.

El segundo tema, de la voluntad de lo real, se refiere al hecho simple de que nuestro sistema de aprendizaje tiene un alto grado de abstracción y que si bien nuestro propósito final es la edificación de obras reales, tal instancia no la encontramos dentro de la actividad académica (por razones obvias, principalmente económicas y cronológicas).

Ocurre por lo tanto el hecho observable que, si bien la producción creativa e innovadora en el interior de esta escuela y de muchas otras es enorme y admirable, sin embargo todavía es insuficiente la influencia de tales producciones en la ciudad real y su calidad de vida.

Debemos por lo tanto cuidar este contacto de nuestro ámbito de reflexión y abstracción con el mundo real por medio de una voluntad de lo real, voluntad que debería estar presente en todas nuestras acciones académicas y que debería reflejarse en aspectos tales como el dominio de la fineza constructiva, el manejo sustentable de las energías que inciden en la temperie (luz, calor, sonido, etc.), el dominio de la estructuración, capacidad de gestión y otros aspectos que forman parte de la generación de la forma, y que por lo tanto no pueden considerarse como agregados a posteriori. Al contrario, ellos forman parte del proceso que conforma la obra, dándole densidad y riqueza.

Podemos concluir entonces que la forma de una obra no es enteramente representable por medios gráficos bi o tridimensionales, ya que su realidad incluye aspectos mucho más complejos que van desde los procesos de gestión que la hacen posible hasta las energías que la hacen perceptible a los sentidos. Llamamos voluntad de lo real a la preocupación por la totalidad de los factores que inciden en la real calidad arquitectónica de la obra construida.

En respuesta a la segunda pregunta que nos interroga sobre cómo enseñar lo antedicho a los jóvenes arquitectos, pienso que debe hacerse por una parte con la gradualidad propia de todo proceso de aprendizaje y por otra con la transversalidad propia del mundo universitario moderno.

Sin embargo, la gradualidad en el oficio de la arquitectura no debe impedir que desde el primer momento el estudiante se encuentre con todo lo medular del oficio. Vale decir, la gradualidad debe tocar la intensidad o la profundidad de los temas y no la completitud del oficio, la cual debe ser encarada desde un primer instante; tal como el crecimiento de cualquier ser vivo y complejo, que no es miembro a miembro sino que consiste en el perfeccionamiento del ser completo.

En este sentido la actividad de taller es fundamental. Si bien en ciertas etapas conviene hacer talleres con acentuaciones en ciertos temas, tales acentuaciones nunca deben ocupar la totalidad de la fundamentación de un proyecto. El taller tiene por objeto reunir todo lo que en la obra se conjuga.

En cuanto a la transversalidad, en nuestro oficio ella tiene varios ejes. Uno de ellos es el de las magnitudes, que van desde la escala del objeto y del edificio, hasta la escala de la ciudad y del territorio. Otro es el de los grados de abstracción que va desde la reflexión teórica hasta la experiencia de la obra construida.

En lo que se refiere a las magnitudes nuestra Facultad de Arquitectura, Diseño y Estudios Urbanos está constituida en la extensión de dicho eje. Se trata ahora de hacer posible al estudiante el acceso a tal variedad dimensional.

En lo que se refiere a los grados de abstracción, una tarea consiste en relacionar en forma estricta el ámbito académico (de la reflexión teórica rigurosa) con el ámbito profesional (de la reflexión experiencial). Tal relación la buscamos en varias instancias como por ejemplo la participación de profesionales de alto nivel en la actividad de taller, la existencia de tesis proyectuales en nuestros posgrados, etc. Estas acciones, unidas al desarrollo de nuestros posgrados nos permitirán, por una parte, elevar el nivel de la reflexión teórica y, por otra, relacionarla vitalmente con la práctica profesional.

Este escrito es un intento por introducir en forma ordenada, en un espacio quizás demasiado reducido, los aspectos a mi juicio más importantes de la formación de los arquitectos.

\section{Aprender y ejercer arquitectura}

Alberto Sato. Decano de la Facultad de Arquitectura y Diseño, Universidad Andrés Bello

En el mundo occidental, la enseñanza sistemática del oficio del arquitecto tiene larga data: en la Real Academia de Arquitectura francesa el célebre Cours d'architecture de François Blondel se inició en 1675.

Desde ese entonces, la licencia para ejercer la profesión, por la responsabilidad civil que conlleva, quedó fuera del ámbito académico y se otorga por distintas autoridades que certifican la capacidad y destrezas del candidato. Dichas autoridades surgieron de distintas realidades históricas y sociales, desde gremios profesionales, municipios, estados, gobiernos centrales, reyes, reinas o príncipes. De esta variedad, el aspecto común que comparten es que se establecen diferencias entre el conocimiento de la disciplina y la autorización para ejercer la profesión.

Un antecedente significativo fue la creación del Institute of British Architects en 1834, elevado a categoría Real por la reina Victoria en 1866, denominándose desde esa fecha, RIBA, a objeto de "...facilitar la adquisición del conocimiento de la arquitectura para la promoción de las distintas ramas de la ciencia relacionadas con ella, y para establecer una uniformidad y respetabilidad de la práctica de la profesión". Es decir, uniformidad y respetabilidad de la práctica y para ello, acordar los modos a través de los cuales se aprende la profesión, habida cuenta que John Ruskin rechazaba la idea que este arte se pudiese enseñar en la universidad (lo que todavía hoy es legítimo preguntarse). La clase para el examen voluntario dictada en la Architectural Association para entrar al RIBA comenzó en 1863, estableciéndose por primera vez el estudio sistemático para la habilitación profesional comprobado por examen dentro de una universidad. En los Estados Unidos, el Massachussets Institute of Technology (MIT) fue la primera institución académica reconocida por la American Institute of Architects para rendir el examen especial en 1865.

Así, el examen universitario fiscalizado se aplicó en muchos países del mundo occidental para el otorgamiento del título habilitante de arquitecto. Pero en nuestro continente latinoamericano, la universidad asumió la responsabilidad del otorgamiento del título profesional sin otra fiscalización externa, y por lo visto presenta una diferencia respecto del resto del mundo. Se podría arriesgar que la causa no resultó de profundas reflexiones teóricas o pedagógicas, sino de las estructuras institucionales del continente. Así, la universidad se constituyó en uno de los principales referentes éticos y morales de la sociedad latinoamericana. De allí que sus responsabilidades fueron mayores que la de transmitir y producir conocimiento. En virtud de esta tradición, la arquitectura se ha mantenido dentro del hermético círculo universitario, una de cuyas consecuencias es que el llamado mundo real aparece a través de su propia mediación. La universidad otorga licencia vitalicia para ejercer la profesión porque no existen instancias de actualización o condiciones de vigencia. El reclamo de muchos sobre la enajenación de la universidad frente a la realidad 
y en particular, de la distancia entre el ejercicio de una profesión y los saberes adquiridos en las aulas señala, al menos, un problema que pone en crisis el modelo actual de enseñanza. En efecto, los conocimientos que se podrían llamar profesionalistas o instrumentales del oficio de arquitecto se introducen en los planes de estudio desde el comienzo de la carrera, alterando el sentido universitario del conocimiento relevante hacia una operatividad que en muchos casos descuida los conceptos sobre los que se basan. En el campo disciplinar, a los conocimientos relevantes se agrega la necesidad de la experimentalidad proyectual como forma de conocimiento. El ejercicio de la profesión, por su parte, exige del aprendizaje de destrezas y capacidades que permitan abordar las exigencias reales del mercado, pero en la universidad, por su propia naturaleza, estos conocimientos se complejizan con reflexiones teóricas que distorsionan su verdadero carácter instrumental. De este modo son dos finalidades distintas y en consecuencia, exigen contenidos y metodologías distintas. El resultado de esta hibridación es que algunos distinguidos arquitectos dan mayor importancia a las influencias personales recibidas en sus años universitarios, antes que a un programa de estudios. En este sentido, está vigente la polémica introducida por Ruskin.

Un período de cinco años -al menos- de vida universitaria es cualitativamente distinto que vivir la calle. También es distinto reproducir en ese ámbito el ejercicio de una profesión: se entiende que la universidad provee de instrumentos para un ejercicio futuro y no por ello dicho ejercicio debe constituirse en modelo pedagógico. Quizás innecesario, pero es importante confirmar que los modos de aprender universitario son, más que un aprender a hacer, es aprender a pensar; más que conocer, trabajar el conocimiento; más que informarse, trabajar la información. De otro modo, es decir, trasladando la realidad práctica a la academia con el deseo de reproducirla, no se asegura que una carrera deba durar cuatro, seis o veinte años como si se tratara de una escuela de aprendices medievales; la universidad pretende que alguna destreza intelectual se aprenda en cuatro o cinco años. Para ello, algunos rasgos y tiempos de la vida necesariamente son alterados, pero también y en especial, los modos de aprender y de enseñar (Morin, 1999). Al decir de Novak-Godwin, la verdadera educación cambia el significado de la experiencia humana. Ésta es la diferencia con la vida, que también enseña y donde también se aprende, pero sin los plazos y la sistematicidad que los años universitarios exigen.
Producir conocimientos / La tarea universitaria es producir profesionales, pero también producir conocimiento. Sin duda, la disciplina se alimenta sistemáticamente con nuevos conocimientos. Mucho más enriquecedor e innovador si dicha alimentación se produce dentro del proceso académico, es decir, si el aprendizaje basado en el problema en la relación entre docentes y alumnos, la búsqueda en equipo y la experimentalidad, dan como resultado nuevos conocimientos con significación y relevancia.

Este proceso introduce un giro radical a los modos de enseñanza tradicional consistentes en la transmisión presencial de un saber que el docente no produjo y que está registrado en textos de fácil acceso. Si bien se sabe que no se puede producir nuevo conocimiento todos los lunes, también es cierto que la universidad tiene la responsabilidad social de producirlo en algún momento y por lo tanto los modelos pedagógicos deben crear las condiciones para ello. Así la arquitectura, que crea conocimientos por la vía experimental y por la búsqueda -la recherche patiente lecorbusierana- sale al encuentro de nuevos paradigmas pedagógicos, de una epistemología que señala que la experimentalidad proyectual basada en problemas y preguntas, constituye un modo de conocimiento y un campo de innovación.

Bibliografía: AA.VV.; El arquitecto: Historia de una profesión. Spiro Kostof, Ed., Ediciones Cátedra, Madrid, 1977. / AA.VV.; Reflections on Architectural Practices in the Nineties. Peter Rowe, Ed., Princeton Architectural Press, Nueva York, 1996. / AA.VV.; The discipline of architecture, Andrzej Piotrowski y Julia Williams Robinsons, Ed., University of Minnesota Press, Minneapolis, 2001. / Ausubel, D., Novak, J. y Hanesian, H.; Psicología educativa: Un punto de vista cognoscitivo. Editorial Trillas, México, 1976. / Dirección de Investigación y Desarrollo Educativo; El aprendizaje basado en problemas como técnica didáctica. Vicerrectoría Académica, Instituto Tecnológico y de Estudios Superiores de Monterrey, s.d. / Morin, Edgar; El Método. El conocimiento del conocimiento. Ediciones Cátedra, Madrid, 1988. / Morin, Edgar; Los siete saberes necesarios para la educación del futuro. Editorial Paidos - UNESCO, Barcelona, 1999. / Novak, J. y Gowin, D.; Aprendiendo a aprender. Ediciones Martínez - Roca, Barcelona, 1988. / Secretaría General de la UIA; Sistema UNESCO-UIA de validación para la formación de arquitectos. Unión Internacional de Arquitectos, 2002.

\section{Dónde enseño arquitectura}

Juan Román. Director de la Escuela de

Arquitectura, Universidad de Talca

La importancia que Josep Quetglas le otorgaba esa tarde en el COAC de Barcelona ${ }^{1}$ al dónde enseño arquitectura, ponía en perspectiva el

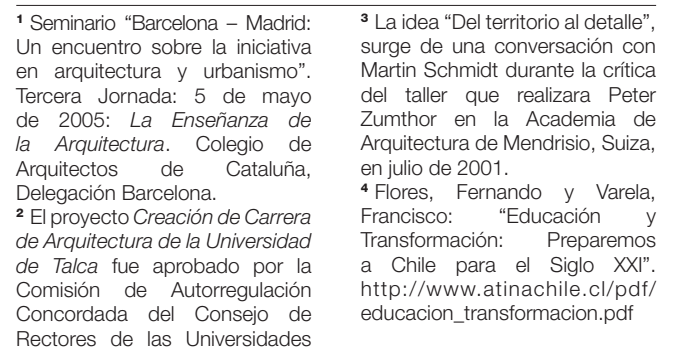

Rectores de las Universidades

Chilenas en julio de 1998

trabajo que, alumnos y profesores de la Escuela de Arquitectura de la Universidad de Talca, habíamos desarrollado en el territorio asociado al valle central de Chile, durante los últimos siete años, al punto de constituir a ese territorio en el soporte de nuestro quehacer.

Vaya entonces aquel grato episodio como introducción a las cinco reflexiones que siguen y que intentan aportar, desde la provincia, a la discusión de la enseñanza de la arquitectura en Chile.

I. El dónde enseño arquitectura agregado al a quién enseño arquitectura constituía el par fundamental para diseñar la enseñanza en una zona en la que nunca existió una escuela de arquitectura; definir el perfil de ingreso del alumno para contraponerlo al perfil de egreso ya contenido en el proyecto de carrera ${ }^{2}$, permitía dimensionar la labor a desarrollar durante los años siguientes.

Igualmente, se estimaba que en el trabajo de aquellas dos preguntas residía la particular manera de hacer, condición que se le reconocía a las respectivas escuelas de arquitectura de las universidades de Chile, Católica de Chile y Católica de Valparaíso, para quedar así apuntando al cumplimiento de la aspiración institucional de constituir a la escuela de Talca en la cuarta escuela de Chile.

II. La casa Marika-Alderton que proyectara Glenn Murcutt y que conociera en 1996 en una publicación, se iba a constituir en un referente a la hora de intentar compatibilizar aquel desfase entre el perfil de alumno y el perfil de egresado. Y es que la capacidad de Murcutt para convertir un encargo corriente en una obra mayor, tenía que ver con las competencias que, en su tránsito entre aquellos dos perfiles, el alumno habría de hacer suyas (Melhuish, 1996).

III. Inscribir el quehacer del arquitecto en un modelo pendular que va del territorio al detalle ${ }^{3}$ ha permitido restarle al territorio su condición de mero contexto, suscribir la condición de profesional no especializado del arquitecto y determinar la organización no departamental de los profesores de la escuela de Talca.

Lo anterior, agregado a la flexibilidad del proyecto de carrera, ha permitido el funcionamiento de esta escuela distante, cuando no existe en el país la cantidad necesaria de profesores para atender los requerimientos de las treinta y tantas carreras de arquitectura, que actualmente funcionan en Chile. IV. Las modificaciones en el sector laboral e institucional empiezan a ser tan rápidas, que las profesiones y carreras de siempre han dejado de ser nichos estables ${ }^{4}$, llevando a reparar en lo vano del esfuerzo que, año a año, estudiantes y profesores de las carreras de arquitectura del país dedicamos 
al proceso de enseñanza - aprendizaje de la proyectación de edificios como única competencia exclusiva de la profesión de arquitecto.

Se combinan aquí las dos responsabilidades sociales que recaen sobre los que tenemos que ver con el diseño de la enseñanza de la arquitectura: asegurar que los egresados cumplan con el rol que la sociedad espera de ellos, cual es la proyectación de edificios, y preparar a esos mismos egresados para que puedan desenvolverse en un trabajo dignamente remunerado.

Lo anterior ha llevado a ordenar el proceso de la enseñanza en Talca en los tres dominios complementarios que siguen:

Operar, que incluye las competencias relativas a la pro-actividad necesaria para el desempeño profesional en un medio competitivo.

Oficiar, que incluye las competencias relativas a la concepción, proyectación y supervisión de la construcción de un edificio, en su definición extensa. Innovar, que incluirá las competencias relativas a informar la transformación del conocimiento en riqueza. Se considera que el diseño de los tres dominios descritos habilita al egresado para insertarse en ámbitos laborales que, siendo distintos al de la proyectación, requieren de la mirada amplia del arquitecto.

V. La conjunción del dónde y del a quién ha dado lugar en Talca a la implementación de una pedagogía basada en la conformación de problemas y la solución de éstos a través del hacer constante e intenso, lo cual, combinado con un cuerpo de profesores con origen en escuelas diversas, cautela la originalidad pretendida por el proyecto.

Finalmente, la analogía del bosque permite consignar la circunstancia de la periferia, que creo necesario aportar a la discusión.

Jüner constituye al bosque, como símbolo, en la patria de la persona libre que decide vivir por sus propios medios, de la persona de acción que opera sin ser percibido, del que tiene una estrategia, del que sabe cuándo actuar, del que comprende los procesos, del que sabe esperar, del que sabe qué esperar (Junger, 1993).

Creo que la Escuela de Arquitectura de la Universidad de Talca tiene que ver con eso; el emboscamiento que Jünger plantea como una opción es para nosotros, los de la provincia, una asumida condición.

Bibliografía: Jünger, Ernst; La emboscadura. Ed. Tusquets, Barcelona, 1993. / Melhuish, Clare; "Architecture \& Anthropology". En Arcbitectural Design 124, Academ Editions, Londres, 1996.

\section{Pensar arquitectura}

Albert Tidy. Director de la Escuela de Arquitectura, Universidad de Chile

La Escuela de Arquitectura de la Universidad de Chile es la más grande, la más antigua y la más diversa del país. Ella está conformada por la escuelas de pregrado, la de posgrado, dos institutos (el de la Vivienda y el de Isla de Pascua) y cuatro departamentos (Construcción, Urbanismo, Historia y Diseño). En su alumnado está reflejado todo el espectro social de profesionales que han proyectado, construido, legislado y administrado la mayoría del quehacer arquitectónico de los últimos 160 años de nuestra nación. Nuestra universidad está consciente del privilegiado rol que le corresponde, como institución pública, de dar cabida a un profesional también diverso, que pueda desempeñarse con éxito en las distintas áreas de ocupación profesional más allá de la actividad estrictamente proyectual.

Nueve premios nacionales de arquitectura y la permanente presencia de profesionales de nuestra escuela en la adjudicación de concursos públicos y privados, además de la ininterrumpida participación en bienales y publicaciones nacionales e internacionales, avalan la ya tradicional excelencia en el campo del diseño arquitectónico. Igualmente existe una actividad tal vez menos protagónica -pero no menos importante- que corresponde a la de los profesionales del ámbito público: de Arica a Punta Arenas y en los más recónditos asentamientos donde exista una Dirección de Obras Municipales encontraremos a alguno de nuestros egresados cumpliendo (a veces de manera heroica) las labores que competen al interés público de la comunidad. Lo mismo ocurre en los ministerios y los programas de ayuda social.

En el ámbito del ejercicio público, también existen actividades asociadas a especializaciones diversas como la academia, la construcción, el diseño urbano, el cálculo estructural, la iluminación, los medios digitales, las tasaciones y la gestión inmobiliaria, por nombrar algunas. Nuestra actividad hoy debe más que nunca tender hacia la especialización con el objeto de expandir el horizonte ocupacional con arquitectos cada vez mejor preparados. Esto debe ocurrir sin perder la cosmovisión disciplinar que faculta a los arquitectos para visualizar el problema puntual dentro del contexto amplio de su formación.

Hoy existen cuarenta y cuatro escuelas de arquitectura en el país. Si bien esta es una situación adversa para las universidades tradicionales, no necesariamente tiene una connotación negativa: al aumentar la competencia se eleva la calidad del producto y en consecuencia, también se eleva el estado de la arquitectura.

Tal vez ésta sea una de las razones que gatilla la demanda cada vez más frecuente para el acceso a instancias de educación avanzada en niveles de postgrado. Durante casi dos décadas nuestro país sufrió las consecuencias del aislamiento cultural. Hoy día gracias a la recuperación de la democracia y la apertura económica, nuestros estudiantes son cada vez más conscientes de pertenecer a un mundo interconectado. La posibilidad de cursar una experiencia académica en el extranjero, gracias a la oferta de becas de perfeccionamiento y créditos blandos, es cada más cercana y posible.

El impacto que ha tenido este fenómeno en la educación de la arquitectura es tremendamente positivo ya que existe una nueva generación de académicos con visiones frescas y renovadas provenientes generalmente desde los centros desarrollados donde se origina la vanguardia, que se suma a la experiencia de los profesores locales consagrados en un proceso de herencia cognitiva y evolución académica.

La educación de la arquitectura, por otra parte, se ha visto enfrentada a cambios importantes durante los últimos diez años. Como profesor de taller he visto cómo los alumnos han emigrado del taller como espacio físico para trabajar desde sus hogares. La aparición de las nuevas tecnologías que obsolecieron al rapidograph y a la regla $T$, hoy permiten que el alumno se comunique en espacios virtuales de diálogo e indagación. Veo con preocupación esta nueva especie de aislamiento, porque una escuela de arquitectura debe ser todo lo contrario: debe haber roce, discrepancia, discusión y diálogo físico.

El campus de nuestra escuela de arquitectura es un lugar privilegiado en pleno centro de la ciudad. Seis centenarias naves paralelas que originalmente constituyeron las pesebreras y dormitorios del regimiento de caballería Cazadores, hoy constituyen las aulas y talleres de los estudiantes. La distribución espacial, no obstante la belleza de sus edificaciones, presenta ciertos problemas de incomunicación ya que cada taller cuenta con su propio espacio físico delimitado y recluido. Cuando pienso en una escuela de arquitectura como edificio, pienso en el Crown Hall de Mies Van der Rohe en el IIT de Chicago o en el Art \& Architecture Building de Paul Rudolph en Yale, donde tuve el privilegio 
de asistir durante dos años como estudiante. Estos lugares son monoespacios donde todos los talleres se articulan bajo un mismo techo. Los estudiantes se mezclan, las fronteras desaparecen y existe espacio para el accidente, el comentario crítico y el intercambio natural de ideas. Pienso que las nuevas tecnologías son perfectamente compatibles con este tipo de estructuras y los talleres sin papel (paperless studios) dejan de tener la tradicional investidura de taller si pierden su rol original de espacio de trabajo comunitario. Es por esto que creo que una universidad no puede limitarse exclusivamente a impartir docencia. Una escuela de arquitectura no puede reducirse a un grupo de académicos destacados que hacen clases y un grupo de alumnos que asiste a éstas como a una cita. Una verdadera escuela de arquitectura es aquella que vive y brilla por la presencia de sus alumnos de manera permanente. Una escuela de arquitectura tiene la voz y opinión de sus académicos, que a través de la investigación formal generan conocimiento. Una escuela de arquitectura no es un lugar de instrucción ni de traspaso de conocimiento, sino un lugar de indagación, cuestionamiento crítico y experimentación.

Una escuela de arquitectura no enseña a hacer arquitectura sino a pensarla. ARQ

\section{Four schools of architecture}

\author{
About the formative process of architects \\ Fuan Ignacio Baixas. Director of the School of \\ Architecture, Universidad Católica de Chile
}

When confronting the problem of the formative process of architects, two main questions come up to my mind:

The first is: Which aspects of the architectural profession should students acquire during their university training, considering that the complexity of this profession demands an expertise that can only be acquired in the course of a lifetime devoted to it, and that in Chile university training does not only lead to an academic degree but also to a professional title? The second is: How can architecture be learned, keeping in mind that it is a profession that combines the utilitarian focus of technical and scientific skills with the purposelessness of artistic occupations.

In answer to the first question, I believe the main thing about the university training of architects is the acquisition of an ethics related to creativity and the determination to transform reality through their work.

An ethics of creativity and the determination to transform reality are two of the main premises on which our school's instruction has been founded. Throughout the years, we have attempted to consolidate and preserve these two principles as the center of the school's spirit.

The first of them has to do with the fact that architectural works are always created in response to a commission and to a particular set of circumstances, i.e. with a given time, place, and potential users in mind, and so they are necessarily original works. This originality aims at the creation of a possible cultural future, and thus belongs to the realm of creative work.

This originality, however, must also have as a result a potentially habitable construction, and so it must consider all of the conditions required to produce that habitability. This is often difficult in an increasingly specialized world where crucial matters are often overlooked, a world that, as Heidegger has put it, is threatened by the concealed essence of modern technique: a production no longer conceived under the model of Greek poiesis but rather as unlimited exploitation of the planet, with all the risk such an attitude entails.

In this world, our university, guided by its commitment to Christian ethics, attempts not only to follow the basic ethical principle of justice, but also to complement it with an ethics based on charity, together with a fostering of the greatest talent given to us by our Creator, creativity destined to the production of beauty, utility, and habitability.

The second of our guiding principles, the determination to transform reality, has to do with the fact that our teaching methods require a great degree of abstraction: even though our ultimate goal is the construction of actual architectural works, our academic activity as instructors does not include it as part of the formative process of future architects due to the time and resources such a commitment would require.

A consequence of this gap between architectural instruction and actual professional practice is that, even though innovation and creativity have a central place in this and other schools, these qualities are not always observable in the architectural production that determines the quality of life in our cities.

We should, therefore, attempt to maintain links to the professional reality and the actual conditions of its exercise in the outside world: our ability to reflect on these conditions and abstract them should always be tempered by a strong determination to transform reality. This determination should be present in all of our academic tasks, and it should have as a result a strong grasp of building techniques in all of their delicate details, a commitment to the use of environment friendly energies; a good command of structuring and management techniques and of all the other aspects involved in the generation of architectural forms. These aspects cannot be taken for granted nor can they be considered as simple "extra features" that can be added later to an architect's training. Much to the contrary, they are part of the productive process of any architectural work, and they give it the richness and density that characterize an accomplished work.

Architectural form thus conceived is not entirely representable through bi- or tri-dimensional models, since it includes many much more complex aspects, including for instance the entrepreneurial impulse that made it possible, or the energies that make it perceptible to the senses. When we speak of our determination to transform reality, we refer to this concern with the totality of the factors that result in the true architectural quality of a constructed work.

In response to the second of the aforementioned questions (how to teach this to architecture students), I believe the two key points are a gradual approach to the transmission of skills that characterizes all educational processes, and the transversal perspective that distinguishes modern university instruction.

The emphasis on the gradual acquisition of skills, however, must not be taken to mean that from the first day of their studies on, students should not face all of the challenges of their future profession in all their complexity. In other words, the familiarization with architecture must be gradual in the sense of a slowly increasing degree of depth and intensity, not in the sense of being incomplete. Just as a living being develops, not as an inarticulate set of separate limbs and joints, but as a whole, architectural training should be all-encompassing from the beginning on.

For this global scope, architectural workshops are fundamental. Even though sometimes it is convenient for a workshop class to focus on a particular aspect of the architectural profession, it should always give room for the consideration of all the aspects that contribute to the realization of a complete architectural work.

As for the transversal perspective, our profession requires it on several levels. One of them 
is the size of the objects it focuses on: from small objects to buildings, from cities to huge extensions of territory; another are the diverse degrees of abstraction with which it is possible to engage in it, from theoretical reflections to concrete constructive work.

In relation to the first of these levels, our Faculty of Architecture, Design, and Urban Studies encompasses all the possible ranges of this problem, and that should make it possible for students to embrace them in their years of training.

The second of these levels (the varying levels of abstraction with which we address the problems of our profession) requires an effort to relate the academic realm (characterized by the rigor of its theoretical reflection) with the professional realm (characterized mainly by reflections derived directly from experience). We attempt to keep these diverse levels related through the participation of highly qualified professionals in our workshops as well as the option of a project-centered thesis as the final task in our Graduate programs. These instances, together with a further development of our Graduate programs, will allow the improvement of the level of theoretical reflection while keeping it closely related to professional practice.

This perhaps too short text is an attempt to present in an orderly manner what I take to be the main aspects of the formative process of architectural professionals.

\section{Learning and practicing architecture Alberto Sato. Dean of the Faculty of Architecture and Design, Universidad Andrés Bello}

In the Western world, the systematic teaching of the architectural discipline dates from 1675 , with François Blondel's famous Cours d'architecture at the French Royal Academy of Architecture. Since then, the permission to practice the profession of architect, due to the civil responsibility it entails, has been granted not by the Academy, but by authorities that assess and certify the adequateness of the candidate's skills.

These authorities emerged from a varied set of historical and social realities, ranging from professional guilds, town councils, states, central governments, kings, queens, and princes. Their common characteristic is that they all differentiate between the knowledge of the discipline and the authorization to practice it.

A significant moment in this history of the profession was the creation of the Institute of British Architects in 1834, which in 1836 received from Queen Victoria the title of Royal.
From then on it has been known as RIBA. From its inception, its goal has been making possible "...the acquisition of knowledge about architecture in order to promote the many branches of science related to it, as well as establishing uniform and respectable standards for the exercise of the profession". This emphasis on unified and respectable standards implied having agreed on the most appropriate ways to teach architecture, even though someone like John Ruskin could still be strongly opposed to the idea that architecture was susceptible of being taught within a university (a position that remains legitimate). In 1863, the Architectural Association was the first academic institution to include in its curriculum a course designed to prepare the voluntary exam needed in order to become a member of the RIBA. That was the first time a university offered a systematic preparation for the professional habilitation through an exam. In the United States, the Massachusetts Institute of Technology -MIT- was the first academic institution to be authorized by the American Institute of Architects to give the special exam in 1865 .

Thus, a certified exam was instituted in many Western countries as a requisite to obtain the professional title of architect. In Latin America, however, in contrast with the rest of the world, it was the Universities that took upon their shoulders the responsibility of granting professional titles, without resource to any external supervising instance. One could perhaps venture the hypothesis that this did not happen as a consequence of deep theoretical of pedagogic reflections, but rather as a result of the state of the institutional structures of our continent. Thus, the university became one of the main ethical and moral references of Latin American societies, and so it was not only responsible for the transmission and production of knowledge. As a consequence of this tradition, architecture has remained enclosed within the narrow circle of the university disciplines, with the effect that its exposure to the so called real world is always mediated by its position as part of the academy. Universities can grant a lifelong permission for the exercise of the profession because no institution is in charge of determining the conditions for the actualization or renewal of this authorization. This model of learning is presently in a state of crisis made evident by the many voices who complain about the alienation of the universities from the rest of reality, and more in particular the enormous distance that separates the skills acquired during university training from the actual demands of professional practice. What can be called "professional" or "instrumental" tools are, in fact, introduced to the students during the first years of their training, inflicting the university's focus on relevant knowledge towards a preoccupation with operational efficiency that too often remains blind to its own conceptual basis. In terms of the discipline's demands, the acquisition of relevant knowledge must be complemented by experience in architectural projects as a form of knowledge. On the other hand, professional practice requires mastering skills and techniques that enable an architect to deal with the actual demands of the market, but in a university the transmission of this know-how is complicated by theoretical reflections that distort its true instrumental character. This is the result of the overlapping and mingling of two different goals, each of which would require its own set of contents and methodologies in order to be effectively transmitted. As a result of this hybrid approach, several important architects declare that their most important experience during their university training was not any particular curriculum but the exposure to some relevant personal influences. In this sense, it is still possible to share Ruskin's opinion about the teaching of architecture.

Spending five years in college is obviously different from, let us say, spending them in the streets. In the same way, it is different to exercise a profession than to study it as part of a university curriculum: the university is supposed to provide tools for a future professional practice, but must not necessarily take that professional exercise as its pedagogic model. It may seem a truism, but we should not forget that university education is less a transmission of ways of doing certain things than a transmission of modes of thinking; thus, it is less about acquiring certain specific sets of knowledge than about figuring out how knowledge works; less than about becoming informed of certain facts, than about understanding the way information works. In other words, if we simply attempt to translate professional practice into the academic setting we could teach it for four, six, or twenty years, as in a medieval apprenticeship training. Instead, what we are supposed to do is transmit in four or five years some intellectual skills, and it is with that goal in mind that we have to transform some features and rhythms of the outside world, but also must continuously transform the ways in which we teach and learn (Morin, 1999). As Novak-Godwin puts it, true education changes the meaning of human experience. This is the difference with life, which 
also teaches us many things, but without the systematicity and the periodic structure that our university years demand.

Producing knowledge / The university's mission is not only to produce professionals, but also to create new knowledge. Doubtlessly, the progress of any given discipline requires this constant production of new knowledge, and it is even better if such a growth takes place as the result of the interaction between teachers and students in an academic context, that is, if the transmission of knowledge through teamwork and experimentation results in new, significant insights about the profession.

This conception of the university's task is radically different from the traditional modes of teaching wherein professors merely pass on a set of notions in the production of which they did not play an active role, and which thus could also be easily acquired through textbooks or other sources. Of course, universities cannot be expected to be producing new knowledge during every single moment of their work, but they are expected to generate it on the long run, and so our ways of conceiving the pedagogical approach for the training of professionals in a given area should contribute to the fulfillment of this goal by creating the conditions it requires. Architecture is a discipline that creates new knowledge through experience and research -as in Le Corbusier's recherche patiente -, and thus it must engage enthusiastically in the search for new pedagogical paradigms, as part of an epistemological approach that acknowledges the fact that the experience of architectural projects based on problems and questions does constitute a mode of innovation and knowledge.

Bibliography: AA.VV.; El arquitecto: Historia de una profesión. Spiro Kostof, Ed., Ediciones Cátedra, Madrid, 1977. / AA.VV.; Reflections on Architectural Practices in the Nineties. Peter Rowe, Ed., Princeton Architectural Press, New York, 1996. / AA.VV. The discipline of arcbitecture, Andrzej Piotrowski and Julia Williams Robinsons, Ed., University of Minnesota Press, Minneapolis, 2001. / Ausubel, D., Novak, J. y Hanesian, H.; Psicología educativa: Un punto de vista cognoscitivo. Editorial Trillas, México, 1976. / Dirección de Investigación y Desarrollo Educativo; El aprendizaje basado en problemas como técnica didáctica. Vicerrectoría Académica, Instituto Tecnológico y de Estudios Superiores de Monterrey, s.d. / Morin, Edgar; El Método. El conocimiento del conocimiento. Ediciones Cátedra, Madrid, 1988. / Morin, Edgar; Los siete saberes necesarios para la educación del futuro. Editorial Paidos - UNESCO, Barcelona, 1999. / Novak, J. y Gowin, D.; Aprendiendo a aprender. Ediciones Martínez - Roca, Barcelona, 1988. / Secretaría General de la UIA; Sistema UNESCO-UIA de validación para la formación de arquitectos. International Union of Architects, 2002.
Where do I teach architecture?

Fuan Román. Director of the School of Arcbitecture,

Universidad de Talca

That evening in the COAC at Barcelona, ${ }^{5}$ the central place given by Josep Quetglas to the question "where do I teach architecture?" helped me to put in perspective the work that we, the students and instructors of the School of Architecture at Talca University, have carried out in the central valley of Chile over the last seven years, to the extent that this site has become the main foundational ground of our academic endeavours

The pleasurable memory of that talk is a good starting point for the following reflections, an attempt to contribute from the region of Chile where I work to the discussion of how to teach architecture in our country.

I. The question "where do I teach architecture?" taken together with the question "to whom do I teach it?" were the two basic foundations starting from which we designed our curriculum for a school that would be situated in an area where architecture had never been taught before. These two questions allowed us to define the profile of our future incoming students and to contrast it with the projected profile of our future alumni, ${ }^{6}$ so as to be able to determine the main tasks to carry out during the following years for the creation of our school.

We also believed that in carefully addressing the problems posed by those two questions, we would be able to design our own particular way of teaching architecture, as it had been already done by schools such as the ones of the University of Chile, the Catholic University of Chile, and the Catholic University of Valparaíso. Thus we could in time become the fourth important school of architecture in the country. II. The Marika-Alderton house designed by Glenn Murcutt, which I got to know through a publication in 1996, served as one of the main referents in the task of bridging the gap between the profile of our incoming students and the projected profile of our alumni. Murcutt's ingenuity in turning a completely ordinary commission into a major work of architecture was the ability that we needed to transmit to our students. (Melhuish, 1996)

III. We conceive the architect's job in a dialectic movement from the territory to the detail. ${ }^{7}$ Thanks to such a conception the site ceases to be conceived as the mere context of an architectural work: this has allowed us to conceptualize architecture as a non-specialized field and in

"I am alluding to the Conference
"Barcelona - Madrid: a Seminar
on Architectural and Urbanistic
Initiative". Third day, May 5
2005: Teaching Architecture.
Architectural Association of
Catalonia, Barcelona chapter.
6 The project Creation of an
Architecture School for the Talca
University was approved by the
Comisión de Autorregulación
Concordada of the Consejo
de Rectores of all Chilean
universities in July 1998

The idea of a dialectic between the territory and the detail had tis origin in a conversation that took place in one of the sessions at the Zunithors workshop at Pe Architecture Academy in 2001

Flores, Fernando y Varela Francisco: "Educación y Transformación: Preparemos itp: / para el siglo $x$ r educacion transfornacion pdf turn generate a non-departmental organization for the faculty of our school.

This, together with the great flexibility of our project for the school, has allowed the good development of this school situated in a distant region of the country, at a moment when there are not enough architecture professors to fully respond to the demands of the more than thirty schools of architecture presently operating in the country.

IV. These days, changes in the professional and institutional market happen at such a fast pace that the typical university professional trainings have ceased to be the stable entities they used to be: ${ }^{8}$ because of this evolution, the model of an architectural training as mere projection of buildings has become obsolete.

This new situation has also transformed the traditional social responsibilities that we as architecture instructors have: on the one hand, making sure that our students are qualified to respond to the demands our society places upon them (being able to project buildings); on the other, training those students so they can have access to a well paid job in the future.

These demands have lead to the organization of our curriculum in the three following domains: Operational know-how, including the acquisition of the necessary skills for an adequate professional performance in a competitive world.

Professional training, including the tools required for the conception, projection and supervision of the construction of a building, understood in the widest possible sense.

Innovation, a set of tools destined to enable the students to transform their knowledge in material wealth.

We believe that a good training in these three domains will give our students the ability to insert themselves in the diverse professional contexts (including, but not limited to, the projection of buildings) that require an architectural perspective.

$\mathrm{V}$. The combined problem of where and to whom de we teach architecture has resulted in a pedagogy that centres in the formulation and solution of problems through intense and persistent work, an approach that, combined with the diverse procedence of our faculty, guarantees the originality of our academic project.

As I final remark, I would like to recall Ernst Jünger's notion of "the woods" as an analogy to our conception of what a school of architecture should be: for him, the woods are a symbol of freedom of the individual living on his or her own means, of the person whose actions are not 
perceived because they do not take place in the open space, the one that knows when to wait and when to act, the one that understands processes and their results, and thus knows what to hope for (Jünger, 1993).

I believe Jünger's metapor is a good image for what we have been trying to do in the School of Architecture at Talca University: for him, life in the woods was an option; for us it is more of an assumed condition.

Bibliography: Jünger, Ernst; La emboscadura. Ed Tusquets, Barcelona, 1993. / Melhuish, Clare; “Architecture \& Anthropology". In Architectural Design 124, Academ Editions, London, 1996.

\section{Thinking architecture}

Albert Tidy. Director of the

School of Architecture, Universidad de Chile

The School of Architecture of the University of Chile is the largest, oldest, and the most diverse in our country, comprising the Graduate and Undergraduate programs, two Institutes (Housing and Pascua Island) and four Academic Departments (Construction, Urbanism, History, and Design). Its diverse body of students accurately represents the broad social scope of professionals that have projected, constructed, managed, and created laws to organize most of the country's architectonic achievements in the last 160 years. Our university is well aware of its privileged role as a public educational institution with the mission of making possible the existence of professionals with a varied and diverse set of skills that will enable them to successfully engage in the various tasks required by our discipline, not limited exclusively to the creation and construction of architectural projects.

Our school's excellence in the domain of architectural design is clearly demonstrated by the Nine National Prizes of architecture granted to alumni of our school, as well as by their constant presence as winners of public and private architectural contests, not to mention their uninterrupted participation in architecture biennials or national and international publications. There is also the less visible -but not less significant- activity of professionals working in governmental offices: from Arica to Punta Arenas, wherever a Municipal Office for the Supervising of Buildings operates, some of our alumni exercise their profession there, contributing in a sometimes heroic effort to the better service of their respective communities.
The same can be said of Ministries and social work programs all over the country.

In addition, there are other ways to exercise our profession in the public domain, such as the academic realm, construction, urban planning, structural planning, lightning, digital media, building appraisals, and real estate, to mention only a few. Our activity in the present should tend more and more towards specialization, so as to further expand the possibilities for practicing our profession while improving the standards of professional training. This should not mean the loss of the particular perspective that characterizes an architect's Weltanschauung, the ability to address concrete problems in the light of the insights gained during the formative years of exposure to an intellectual tradition.

There are today forty four schools of architecture in our country. This evidently makes the work of the older institutions harder, but it is not necessarily a negative development: a more competitive market encourages a constant search for better educational quality, and thus in the long run, produces better architecture.

This may be one of the reasons behind the increasing demand for Graduate education. After nearly two decades of cultural isolation, the recovery of democracy has brought along with it a greater economic mobility, and our students are nowadays aware of the fact that they are part of a tightly interconnected world. Graduate studies abroad have also become much more easily accessible thanks to the abundance of scholarships or credit loans specially designed for students.

The consequences of these changes for architectural education have been extremely positive: our universities house nowadays a new generation of academics that have brought with them fresh, innovative ideas, often derived from their contact with the more developed centers where avant-garde thought is being produced. This new generation of academics now interacts fruitfully with their older, more experienced, locally trained pairs, in a process of constant cognitive and pedagogic exchange.

Architectural education, on the other hand, has suffered important changes over the last ten years. As a workshop professor, I have seen students abandon the physical space of the workshop in order to work at their homes. The emergence of new technologies that have put the work with ink pens and T-shaped rulers far behind allows students to communicate and carry out research through virtual space. I am personally somewhat troubled about this evolution, since it brings along a greater isolation, the contrary of what an architecture school should be, including friction, discrepancy, discussion, and physical as well as intellectual interaction.

Our school's campus is a privileged place, located at the center of Santiago. Six parallel blocks that originally served as mangers and dormitories for the Hunters military regiment of the Cavalry have been adapted as the classrooms and workshops for our students. This layout, in spite of the beauty of the buildings, has produced some communication problems, since all of our workshops have been confined to their own isolated space. In contrast with this arrangement, one could invoke the buildings that housed architecture schools such as Mies Van der Rohe's Crown Hall at Chicago's IIT, or Paul Rudolph's Art \& Architecture Building at Yale, a place that I had the luck of getting to know and use for two years as a student. These buildings are single spaces where all of the workshops can take place under the same roof. Students can intermingle freely, boundaries disappear, and critical commentary as well as intellectual exchange can take place naturally. I believe new technologies are perfectly compatible with this kind of structures, and perhaps we should keep in mind that the so-called paperless studios may lose their original condition of workshops if they cease to be a space of communal work.

A university's mission is not limited to teaching. A school of architecture should not be just a group of renowned academics that teach great classes and a group of students that sits still while listening to them. A true school of architecture acquires its brilliance thanks to the active presence of its students. A true school of architecture cannot but listen to the voice of its academic body, which constantly creates new knowledge through research. A school of architecture is not just a place for instruction, but a place for intellectual enquiries, critical questionings and experimentation.

A school of architecture does not only teach how to practice architecture but how to rethink it. ARQ 